\title{
Current progress of adeno-associated-virus-based therapy for the treatment of cystic fibrosis in humans
}

\author{
R. Bryan Sutton, PhD
}

\section{INTRODUCTION}

For almost 40 years, gene therapy has been hailed as a cure for all of man's ailments. The potential of gene therapy is similar in many ways to that of nuclear fusion as an infinite, cheap energy source. Grandiose claims have been made over the last several decades without any clear results. However, like the promise of nuclear fusion, the complexities of the problem and various regulatory setbacks have slowed the implementation of this promising therapy. Indeed, the ability to precisely edit an errant locus in a medically crucial gene is, indeed, an exceedingly powerful therapy, and the dawn of the $21^{\text {st }}$ century has ushered in multiple new techniques capable of achieving this feat. These revolutionary techniques have opened up a wide variety of new possibilities to treat previously intractable diseases at the most fundamental level.

\section{HISTORY OF GENE THERAPY}

Gene therapy was a pipe dream in the 1960s. One of the first statements alluding to gene therapy was offered by Dr. Marshall Nirenberg, Nobel Laureate in Physiology 1968. In a talk presented at the Research Corporation Award Dinner in 1967, Nirenberg mused about how "genetic surgery" could potentially be used to cure a multitude of human diseases. ${ }^{1}$ In the same speech, however, he recognized that the method could be abundantly dangerous. In 1990, a fouryear-old girl named Ashanti DeSilva became the first

Corresponding author: Roger Sutton

Contact Information: Roger.b.sutton@ttuhsc.edu DOI: 10.12746/swrccc.v6i23.461 successful recipient of gene therapy. ${ }^{2}$ In Ashanti's case, a corrected version of the adenosine deaminase (ADA) gene was used to treat severe combined immunodeficiency syndrome (SCID) by introducing the normal form of the ADA gene into ex vivo white blood cells, which were then reinfused. While Ashanti showed only a partial restoration of ADA function due to the limitations of the ex vivo technique that was used, she is currently alive and well with her condition. However, not all early recipients of gene therapy were as fortunate. In 1999 Jesse Gelsinger received a gene encoding ornithine transcarbamylase via an adenovirus vector. Four days after the therapy, Jesse died from a massive anaphylactic reaction. ${ }^{3}$ The mistakes made in the Gelsinger case fundamentally changed how universities conduct clinical trials that involve gene therapy and how they admit human patients into future clinical studies. ${ }^{4,5}$ Indeed, gene therapy research was set back by a decade as a result of the Gelsinger case. In the intervening years since Jesse's death, patient acceptance policies for clinical trials have been clarified, and more effective virus-mediated gene transfer methods have been developed. Adeno-associated virus (AAV) gene transfer is proving to be one of the most effective tools for genetic editing.

Of course, repairing genomes via an exogenous AAV suggests a number of questions concerning the efficacy of gene therapy. For example, is one treatment with virus sufficient or do the introduced changes propagate to subsequent daughter cells? In other words, are the changes to cells reliably inherited? Another question is how extensive the repair may be. Are the repaired cells and their daughter cells as good as wild type cells or will there be some residual damage no matter how much repair is attempted? The real extent of these questions remains to be answered. 


\section{THE MECHANISM OF AAV-MEDIATED GENE TRANSFER}

The AAV virus is a helper-dependent parvovirus with an approximately $4.7 \mathrm{~kb}$ genome. The single-stranded, linear DNA genome encodes only two open reading frames (ORFs). One ORF encodes proteins for replication, while the other ORF encodes proteins to make capsid. In this technique, the DNA of interest is grafted into the AAV genome. Only the inverted terminal repeats (ITR) are required for successful integration. Once the re-engineered AAV particle infects a target cell, it inserts the new gene in a site-specific manner into a non-coding region on chromosome 19 in humans. From there, the new gene can produce the corrected protein. Over 100 different serotypes of AAV have been characterized. Interestingly, different serotypes of AAV have a preference for different tissues. ${ }^{5}$ For example, AAV1, AAV6, and AAV7 tend to transduce muscle tissue with high efficiency. AAV8 and AAV9 tend to have the highest level of hepatocyte transduction. ${ }^{6} \mathrm{AAV} 4$ prefers pulmonary tissue. ${ }^{7}$

\section{AAV-MEDIATED PULMONARY DELIVERY}

The use of AAV-mediated gene therapy is well suited for pulmonary delivery for a number of reasons. First, pulmonary tissue is well vascularized for access to circulating blood supply and subsequent systemic delivery. The use of nebulizers to deliver measured dosages of recombinant virus to pulmonary tissue also has great advantages relative to systemic delivery. However, AAV-mediated transgene expression can be limited in the host because of low-efficiency viral integration and normal pulmonary epithelium turnover. Readministration of the virus to increase integration rates and to overcome tissue turnover can result in an immune reaction to further depress viral effectiveness.

\section{THERAPIES DIRECTED TOWARD CYSTIC FIBROSIS}

Cystic fibrosis (CF) is an autosomal recessive disease caused by mutations in the gene encoding a pulmonary chloride channel, the CF transmembrane conductance regulator protein (CFTR). From a clinical perspective, CF is characterized by chronic airway obstruction, an inability of the pancreas to secrete pancreatic enzymes, and abnormally high sweat electrolyte concentration. Typically, the airways of the lung are cleared of larger airborne particles (dust, smoke, bacteria) via the mucociliary escalator. In CF, increased mucus viscosity impedes the clearance of particles from pulmonary airways. The added viscous medium in the lungs results in inflammation and polymorphonuclear neutrophilic leukocyte infiltration. As a result, CF patients experience chronic airway infections by Pseudomonas aeruginosa and Staphylococcus aureus. Current therapies focus on alleviating the symptoms of the disease by enzymatic thinning of the mucus (mucolytics), intravenous antibiotics, and bronchodilators. Clearly, a more direct therapy is needed. In many ways, CF is an ideal disease to correct via gene therapy. The disease is caused by the loss of function of a single gene product, the wild-type CFTR gene is small enough to fit into a viral vector for delivery, and gene transfer could be conducted via aerosol delivery. Initial Phase 1 trials of AAV2-CFTR were conducted in $1996 .{ }^{8}$ Results of this study concluded that gene transfer rates were slowed by excess mucosal secretions occluding viral binding sites in the bronchial epithelium. In addition, the host's own innate immune response inhibited productive viral infection. ${ }^{9}$ However, despite the low transfection rates, primary nasal cells that were assayed for CFTR mRNA expression and chloride channel activity showed promising positive results. ${ }^{10}$ Phase 2 was a double-blinded, placebo-controlled study to assess the efficacy of AAV2-CFTR when introduced directly into the airways of patients. In this study, gene transfer was apparent in many of the patients, but productive gene expression was not observed. The lack of measurable CFTR activity in the target tissue was likely due to inefficient intracellular processing. Follow up Phase 2 trials with patients receiving AAV2-CFTR failed to show a significant improvement in $\mathrm{FEV}_{1} \cdot{ }^{11,7}$

\section{FuturE OF GENE THERAPY TO TREAT CFTR}

Adeno-associated virus (AAV)-mediated gene transduction is quickly becoming one of the safest 
and most reliable methods for genetic modification. Currently, there are two FDA-approved viralmediated delivery products for use in the USA. Luxturna (voretigene neparvovec) is an AAV-based drug being used to treat biallelic RPE65 mutationassociated retinal dystrophy. Imlygic (talimogene laherparepvec) is a modified herpes simplex virus Type 1-based drug being used as an oncolytic to treat advanced melanoma. ${ }^{12}$ However, for CFTR, small molecule therapies will likely dominate the treatment regime for the near future. Perhaps as methods are developed that can overcome the low rates of infection and the hostile immunological environment in the diseased lung, AAV-mediated therapy may once again prove useful. Advances in nonviral techniques of genetic editing, such as CRISPR/Cas9, may be the key to the future of therapy for CFTR.

Keywords: Cystic fibrosis, CFTR, adeno-associated virus, gene therapy

Financial support: This publication was supported in part by The National Institute of Arthritis and Musculoskeletal and Skin Diseases of the National Institutes of Health under Award Number R01AR063634. The content is solely the responsibility of the author and does not necessarily represent the official views of the National Institutes of Health.

From: Department of Cell Physiology and Molecular Biophysics, Texas Tech University Health Sciences Center, Lubbock, TX

Submitted: 3/28/2018

Conflicts of interest: none

This work is licensed under a Creative Commons Attribution-ShareAlike 4.0 International License.

\section{REFERENCES}

1. Nirenberg W. Will Society Be Prepared? 1966: Research Corporation for Science Advancement. Research Corporation Award Dinner.

2. Bordignon C, Notarangelo LD, Nobili N, et al. Gene therapy in peripheral blood lymphocytes and bone marrow for ADA-immunodeficient patients. Science 1995;270:470-75.

3. Sibbald B. Death but one unintended consequence of gene-therapy trial. CMAJ 2001;164:1612.

4. Kim WO. Institutional review board (IRB) and ethical issues in clinical research. Korean J Anesth 2012;62:3-12.

5. Zincarelli C, Soltys S, Rengo G, Rabinowitz JE. Analysis of AAV serotypes 1-9 mediated gene expression and tropism in mice after systemic injection. Mol Ther 2008;16:1073-1080.

6. Schultz BR, Chamberlain JS. Recombinant adeno-associated virus transduction and integration. Mol Ther 2008;16: 1189-1199.

7. Moss RB, Milla C, Colombo J, et al. Repeated aerosolized AAV-CFTR for treatment of cystic fibrosis: a randomized placebo-controlled phase 2B trial. Hum Gene Ther 2007;18: 726-732.

8. Flotte T, Carter B, Conrad C, et al. A phase I study of an adeno-associated virus-CFTR gene vector in adult CF patients with mild lung disease. Hum Gene Ther 1996;7:1145-1159.

9. Loring HS, ElMallah MK, Flotte TR. Development of rAAV2CFTR: History of the First rAAV Vector Product to be Used in Humans. Hum Gene Ther Methods 2016;27:49-58.

10. Flotte TR, Schwiebert EM, Zeitlin PL, Carter BJ, Guggino WB. Correlation between DNA transfer and cystic fibrosis airway epithelial cell correction after recombinant adeno-associated virus serotype 2 gene therapy. Hum Gene Ther 2005;16:921-928.

11. Moss RB, Rodman D, Spencer LT, et al. Repeated adenoassociated virus serotype 2 aerosol-mediated cystic fibrosis transmembrane regulator gene transfer to the lungs of patients with cystic fibrosis: a multicenter, double-blind, placebo-controlled trial. Chest 2004;125:509-521.

12. FDA. Approved Cellular and Gene Therapy Products. 2018. https://www.fda.gov/BiologicsBloodVaccines/Cellular GeneTherapyProducts/ApprovedProducts/default.htm. 